\title{
Isolated Recurrence of Intracranial and Temporal Bone Myeloid Sarcoma -Case Report-
}

\author{
Mineko MURAKAMI, ${ }^{1}$ Takeshi UnO, ${ }^{1}$ Hiroshi NAKAGUCHI, ${ }^{1}$ \\ Shoko M. YAMADA, ${ }^{1}$ Katsumi HOYA, ${ }^{1}$ Kazuto YAMAZAKI, ${ }^{2}$ \\ Yasuo ISHIDA, ${ }^{2}$ and Akira MATSUNO ${ }^{1}$
}

Departments of ${ }^{1}$ Neurosurgery and ${ }^{2}$ Pathology, Teikyo University, Chiba Medical Center, Ichihara, Chiba

\begin{abstract}
A 52-year-old man presented with a rare case of isolated recurrence of myeloid sarcoma (MS) manifesting as rapidly growing intracranial and temporal bone masses 5 years after complete remission (CR) of acute myeloid leukemia (AML) (M2 in the French-American-British classification). Magnetic resonance imaging and computed tomography of the head revealed enhanced mass lesions on the superior aspect of the right petrous bone and within the right mastoid air cells, extending into the right middle ear cavity without bone destruction. Peripheral blood testing found no evidence of relapse. As the right temporal bone mass showed significant growth into the right temporal lobe over one month and diagnostic tissue was not available, he was admitted to our department for biopsy. On admission, blood testing showed relapse of AML and tumoral hemorrhage in the right temporal lobe. Emergent evacuation of the hematoma and biopsy of the right temporal lobe tumor was performed, resulting in a diagnosis of MS. Chromosomal analysis of the peripheral blood identified $t(8 ; 21)(q 22 ; q 22)$ abnormality. Before initiation of anti-AML chemotherapy, tumoral bleeding occurred again and he died 12 days after the operation of brain herniation. Autopsy revealed leukemia cell infiltrations in the perivascular region of the peritumoral brain parenchyma associated with hemorrhage, indicating vascular damage caused by leukemic cell infiltration from MS induced cerebral hemorrhage. Although relapse after 5 years of CR is uncommon in patients with AML, the possibility of MS recurrence must be considered.
\end{abstract}

Key words: acute myeloid leukemia, central nervous system, myeloid sarcoma, temporal bone, leukemia

\section{Introduction}

Myeloid sarcoma (MS), previously known as granulocytic sarcoma or chloroma, is a rare condition characterized by the occurrence of one or more tumor masses, consisting of immature myeloid cells at an extra-medullary site. ${ }^{10)} \mathrm{MS}$ may develop with acute myeloid leukemia (AML) or other myeloid neoplasms, such as myeloproliferative neoplasm and myelodysplastic syndrome. Most often, MS is found concurrently in a patient with AML, but may precede the appearance of blood or bone marrow disease, and can be the initial manifestation of relapse of previously treated AML in remission. The incidence of MS with AML is 1.4-8.0\%, and usually involves the skin, bone, or lymph nodes, but can affect any site. ${ }^{8,10,14)}$ Central nervous system (CNS) manifestations of MS are rare, based largely on case reports and/or technically dated contributions.

We treated an adult patient with intracranial and tem-

Received March 24, 2011; Accepted May 20, 2011 poral bone MS, who initially presented with isolated recurrence of AML after complete remission (CR) for 5 years. The systemic AML subsequently relapsed, and tumoral bleeding shortened the patient's survival. Although intracranial hemorrhages are not uncommon in AML patients, hemorrhagic CNS MS is very rare. The clinical, radiological, and histological findings of MS are discussed briefly.

\section{Case Report}

A 52-year-old man visited the outpatient otolaryngology clinic in September 2007 with a 1-month history of vertigo and right hearing disturbance, and right acute facial palsy and postauricular pain persisting for 10 days. He had a past history of AML, subtype M2 in the French-AmericanBritish (FAB) classification, which had been in CR for 5 years post-chemotherapy. Peripheral blood tests showed white blood cell (WBC) count, $9.9 \times 10^{9} / 1$ with $0 \%$ blasts; platelet count, $173 \times 10^{9} / \mathrm{l}$; and hemoglobin, $17.5 \mathrm{~g} / \mathrm{dl}$. Ini- 

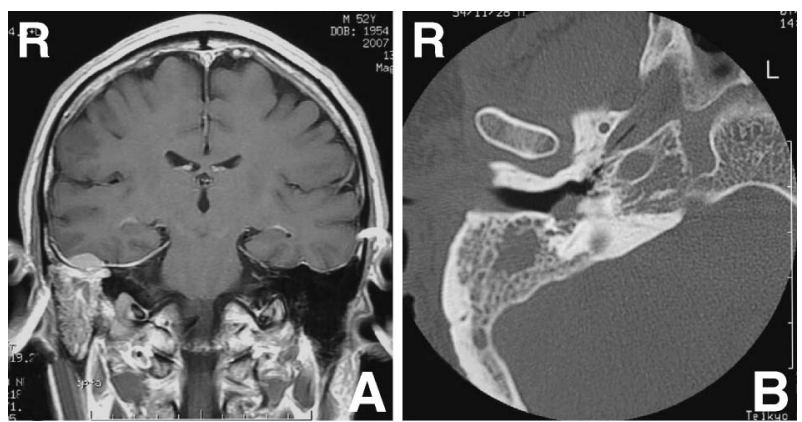

Fig. 1 A: $T_{1}$-weighted magnetic resonance image with contrast medium taken in October 2007 showing a well-defined tumor mass with homogeneous enhancement on the superior aspect of the right petrous bone, broadly contacting the dura, with dural enhancement. The right mastoid cavity is occupied by an irregular enhanced mass. B: Computed tomography scan of the right temporal bone showing the mass extending into the middle ear cavity with little erosion of the bone structure.

tially, he was treated for Hunt's syndrome, but his symptoms showed no improvement.

Brain magnetic resonance (MR) imaging in October 2007 revealed a well-defined mass on the superior aspect of the right petrous bone and opacification of the right mastoid air cells. $\mathrm{T}_{1}$-weighted MR imaging with contrast medium showed a temporal bone mass with irregular enhancement and an intracranial mass with strong, homogeneous enhancement, broadly contacting the dura with dural enhancement, indicating an intracranial, extraaxial mass (Fig. 1A). Computed tomography (CT) of the right temporal bone showed soft tissue in the middle ear cavity with little erosion of the bone structure (Fig. 1B). The differential diagnosis included mastoiditis, metastatic tumor, lymphoma, and atypical meningioma.

Repeated otoscopic examination revealed significant growth of the soft tissue mass in the right middle ear cavity that extended to the external auditory canal over 1 month. Biopsy of the mass in the right auditory canal was non-diagnostic because the specimen was small and hemorrhagic. Brain MR imaging taken in November 2007 showed significant enlargement of the right temporal lobe mass with perifocal edema (Fig. 2A). Peri- and intratumoral hemorrhages were suggested as slightly hyperintense areas on $\mathrm{T}_{1}$-weighted images and hypointense areas on $\mathrm{T}_{2}$-weighted images around the lesion (Fig. 2B, C). Since the patient complained of severe right temporalgia, and tissue diagnosis was unavailable, he was admitted to our department.

On admission, blood tests indicated AML relapse: WBC count, $62 \times 10^{9} / 1$ with $90 \%$ blasts; platelet count, $15 \times 10^{9}$ /l; and hemoglobin, $16.4 \mathrm{~g} / \mathrm{dl}$. Coagulation studies showed: fibrinogen, $366 \mathrm{mg} / \mathrm{dl}$; and international normalized ratio of prothrombin time, 1.03. He suddenly became stuporous, and his right pupil became fixed and dilated, on the day after admission. Head CT showed extra-tumoral hemorrhage in the right temporal lobe with uncal herniation, and a crescent-shaped high density area in the
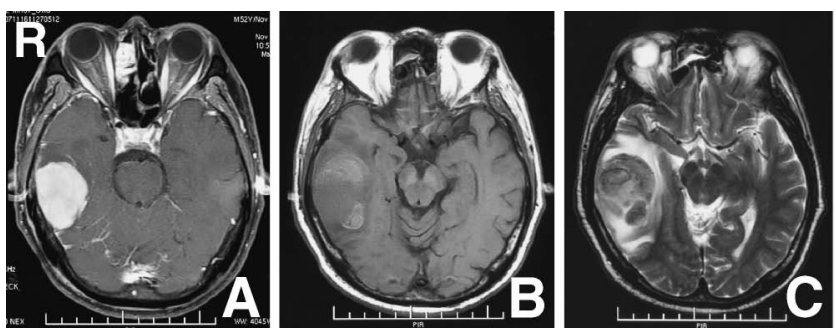

Fig. $2 \mathrm{~T}_{1}$-weighted magnetic resonance image with contrast medium taken in November 2007 showing significant enlargement of the right temporal lobe tumor mass (A), with peritumoral hemorrhages appearing as slightly hyperintense areas on the $T_{1}$-weighted image (B) and hypointense areas on the $T_{2^{-}}$ weighted image (C) around the tumor.

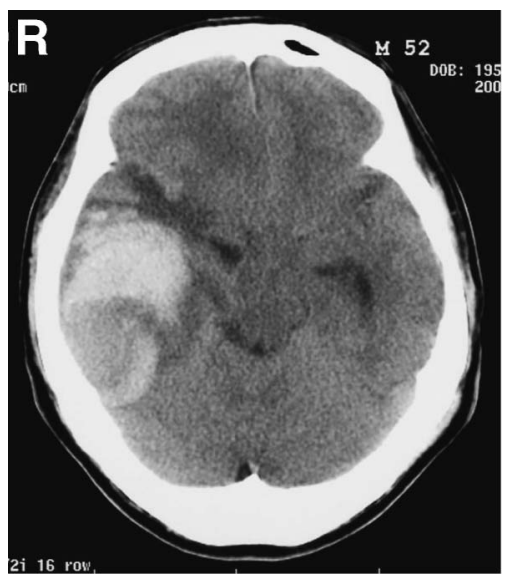

Fig. 3 Computed tomography scan without contrast medium taken on the day after admission showing extra-tumoral bleeding in the right temporal lobe with uncal herniation. The crescent-shaped high density area in the peripheral rim of the tumor indicates intra-tumoral hemorrhage.

peripheral rim of the tumor indicated intra-tumoral hemorrhage (Fig. 3). Emergency biopsy of the intracranial tumor and evacuation of the hematoma via decompressive craniotomy was performed with platelet transfusion procedure.

The tumor was macroscopically firm, grayish, and hemorrhagic. Histological examination demonstrated a monotonous population of atypical mononuclear cells with high nuclear:cytoplasmic ratios, irregular nuclear contours, prominent nucleoli, and coarse chromatin. Tumor cell immunostaining was positive for myeloperoxidase and negative for CD3, CD4, CD8, CD20, and CD30, consistent with MS (Fig. 4). The tumor MIB-1 index was $80 \%$. Cytogenetic studies of peripheral blood revealed that the patient had a $44, \mathrm{X}-\mathrm{Y}$ karyotype and a $\mathrm{t}(8 ; 21)(\mathrm{q} 22 ; \mathrm{q} 22)$ translocation.

Postoperatively, his mental status recovered fully. Systemic chemotherapy was planned and platelet transfusion treatment was repeated, but he again became stuporous because of tumor enlargement and massive tumoral 

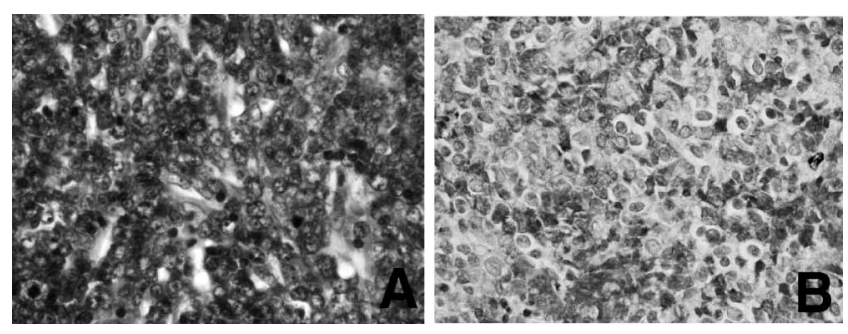

Fig. 4 Photomicrographs of the tumor demonstrating diffuse monotonous atypical mononuclear cells with high nuclear: cytoplasmic ratios (A: hematoxylin-eosin stain, original magnification $\times 20$ ), and immunopositivity to myeloperoxidase (B: immunohistochemical stain, original magnification $\times 20$ ).
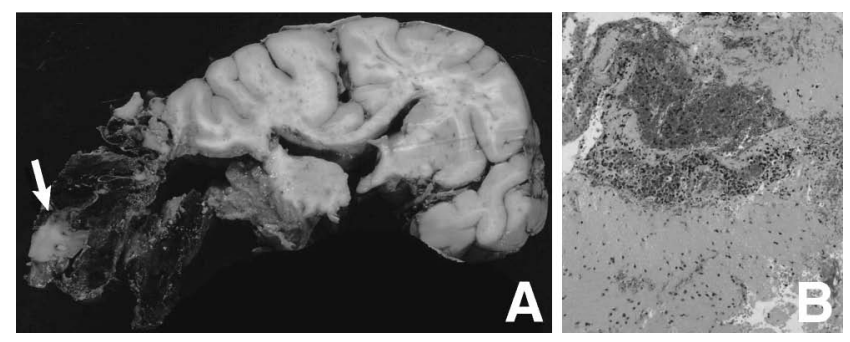

Fig. 5 Autopsy findings. A: Photograph of the brain showing myeloid sarcoma in the right temporal lobe (arrow), associated with subcortical hematoma. B: Photomicrograph of the peritumoral brain parenchyma showing leukemia cell infiltrations in the perivascular region with hemorrhages. Hematoxylineosin stain, original magnification $\times 10$.

rebleeding in the right temporal lobe 8 days postoperatively. He died 12 days postoperatively of brain herniation.

Autopsy revealed systemic relapse of AML and MSs in the right temporal lobe, dura mater, and right temporal bone. Leukemia cell infiltrations were observed in the perivascular region of the peri-tumoral brain parenchyma with hemorrhage, suggesting that the vascular damage caused by leukemic cell infiltrations from MS induced subcortical hemorrhage (Fig. 5). Leukemia cell infiltrations were also observed in the heart, bilateral lungs, esophagus, liver, spleen, bilateral kidneys, prostate, urinary bladder, spinal bone marrow, and systemic lymph nodes. There was no evidence of disseminated intravascular coagulopathy (DIC) in the lungs and kidneys.

\section{Discussion}

Recently, analysis of 92 adult MS patients found that $27 \%$ of patients presented with de novo MS, 35\% had simultaneous AML or other myeloid neoplasm, and $38 \%$ had a previous history of AML or other myeloid neoplasms. ${ }^{10}$ ) The commonest sites of MS presentation were the skin $(28.2 \%)$, lymph node $(16.3 \%)$, testis $(6.5 \%)$, and intestine (6.5\%). ${ }^{10)}$ CNS manifestations accounted for $3.25 \%$ of the cases, so intracranial MS involvement is rare in clinical practice.

The exact pathogenesis of intracranial MS remains un- clear. Leukemic cells do not directly penetrate the capillaries of the brain parenchyma. Possibly the leukemic cells may migrate from the affected bone marrow of the skull via the Haversian canals, infiltrate the dura, and then pass to the subarachnoid space and Virchow-Robin spaces via the perivenous adventitial tissue connecting the dura and the subarachnoidal space. After disruption of the pial-glial barrier in the Virchow-Robin spaces, parenchymal infiltration can result. ${ }^{9,12}$ Unusual manifestations of extraand intracranial locations of MS have been reported..$^{9,17,18)}$ In our case, we speculate that the marrow space of the petrous bone was first involved by leukemic cells, then tumor mass developed and extended to the mastoid cavity and intracranial space.

MS has been described in association with various chromosomal abnormalities such as $\mathrm{t}(8 ; 21)(\mathrm{q} 22 ; 22)$ translocations, mixed lineage leukemia rearrangements, trisomy 8 , and inversion $16 .^{10,12,14)}$ In particular, $\mathrm{t}(8 ; 21)(\mathrm{q} 22 ; 22)$ is regarded as a recurrent aberration in MS. However, such translocations have been detected in MS more often in pediatric patients and/or at the orbital level, and in patients who clinically presented with AML. ${ }^{10)}$ The true incidence of isolated intracranial MS with this translocation in adults is unknown because of its rarity, and largescale studies are unavailable.

Imaging characteristics of intracranial MS are nonspecific. MR imaging shows the lesions as isointense or hypointense compared with normal gray matter on $\mathrm{T}_{1}$ and $\mathrm{T}_{2}$-weighted images, with marked homogeneous contrast enhancement. ${ }^{5,12)}$ If the bone is affected, MS lesions appear lytic rather than sclerotic. ${ }^{2,6)} \mathrm{MR}$ imaging reveals the exact extent of the tumor and potential invasion to the surrounding structures well. However, in the absence of an associated hematologic disorder, the lesions are difficult to differentiate only on the basis of imaging findings from other tumors such as metastases, lymphomas, or inflammatory granulomas, and dural based lesions can mimic meningiomas. ${ }^{4,12)}$ Although the imaging appearance is nonspecific, MS should be carefully considered if tumor masses are discovered in a patient with myeloid neoplasms.

The clinical symptoms of intracranial MS depend on the localization of the lesions. The symptoms of temporal bone MS are hearing loss, vertigo, facial palsy, tinnitus, ear fullness, otalgia, retroauricular swelling, and external auditory canal swelling, mimicking otomastoiditis or otitis externa. ${ }^{2,6)}$ Not only MS but also infiltration of leukemic cells, hemorrhages, and infections of the ear may result in otologic symptoms in leukemic patients. ${ }^{2}$

The presence of isolated MS recurrence generally heralds systemic AML relapse. Review of 24 cases of isolated MS recurrence after prior AML found that the mean interval until bone marrow recurrence was 7 months (range, 1-19 months), ${ }^{1)}$ so the diagnosis of MS should be considered as synonymous with AML. The use of systemic antiAML chemotherapy immediately after the diagnosis was associated with a significantly lower probability of developing AML and prolonged survival. ${ }^{15,20)}$ The optimal therapy for patients with intracranial MS has not been determined due to its rarity. Treatment for intracranial MS 
may include surgical excision and external decompression to relieve neurological symptoms. The addition of radiotherapy to systemic chemotherapy was associated with prolonged survival and should be considered. ${ }^{11,12,14)}$

Spontaneous intracranial hemorrhages were the major cause of immediate death in patients treated with antileukemia therapy. ${ }^{13)}$ Thirty (15.5\%) of 194 patients with acute nonlymphoblastic leukemia had intracerebral hemorrhage diagnosed by CT or autopsy. ${ }^{19)}$ The risk factors of intracranial hemorrhage were leukocytosis (more than $10 \times$ $10^{9} / \mathrm{l}$ ), DIC, and thrombocytopenia (less than $20 \times 10^{9} / \mathrm{l}$, in the absence of coagulopathy or an associated platelet dysfunction). Our patient with leukocytosis $\left(62 \times 10^{9 / 1}\right)$ and thrombocytopenia $\left(15 \times 10^{9} / \mathrm{l}\right)$ without DIC, abnormalities that suggested possible hemorrhagic complications. However, hemorrhagic MS in the CNS is extremely rare. A previous patient presented with acute promyelocytic leukemia (M3 in the FAB classification), coagulation abnormalities, and hemorrhagic MS in the cerebellum. ${ }^{3)}$ The larger size of leukemic myeloblasts makes circulation through small vessels more difficult and leads to greater blood viscosity, leukostasis, vascular dilation, tissue hypoxia, and vascular damage, and eventually induces hemorrhage. .7) In our case, leukemia cell infiltrations associated with hemorrhage were observed in the perivascular region of peritumoral brain parenchyma in autopsy studies, suggesting that the vascular damage caused by leukemia cell infiltrations from MS induced fatal intracerebral hemorrhage.

The prognosis for patients with MS is unfavorable. Sixty (89.5\%) of 67 patients for whom follow-up data were available died of the disease. ${ }^{10)}$ The mean survival times of the patients who underwent chemotherapy, surgery, and radiotherapy were 7.1 months, 36 days, and 1 week, respectively. There were no significant differences in terms of survivor incidence between the group with de novo tumor and the group with concomitant or previous hematological disorder. Interestingly, all seven survivors achieved CR following first-line therapy, and bone marrow transplantation resulted in longer survival than conventional therapy. Overall survival at 48 months was $76 \%$ in transplanted patients, but $0 \%$ in those who did not undergo transplantation. Based on these results, the authors strongly recommended that MS patients should undergo high-dose therapies as front-line approach, since this seems to be their only chance to achieve CR and possibly cure the disease.

The majority of AML patients who achieve CR with initial chemotherapy eventually relapse, most often within the first 3 years. Relapse after 5 years of CR is infrequent, occurring in about $0.8 \%$ of all patients who achieved CR or $1.2 \%$ of all relapsed patients. ${ }^{16)}$ Since few patients relapse after achieving CR for more than 5 years, such patients can be considered potentially cured. However, the possibility of MS recurrence must be considered in leukemic patients. Early recognition of MS is the most important step, and the best results may be achieved with immediate induction of anti-AML therapies.

\section{References}

1) Byrd JC, Weiss RB: Recurrent granulocytic sarcoma. An unusual variation of acute myelogenous leukemia associated with 8;21 chromosomal translocation and blast expression of the neural cell adhesion molecule. Cancer 73: 2107-2112, 1994

2) Chang K, Kim D, Jun B, Park Y: Temporal bone myeloid sarcoma. Clin Exp Otorhinolaryngol 2: 198-202, 2009

3) Fukushima S, Terasaki M, Tajima Y, Shigemori M: Granulocytic sarcoma: an unusual complication of acute promyelocytic leukemia causing cerebellar hemorrhage. Case report. J Neurosurg 105: 912-915, 2006

4) Guermazi A, Lafitte F, Miaux Y, Adem C, Bonneville JF, Chiras J: The dural tail sign-beyond meningioma. Clin Radiol 60: 171-188, 2005

5) Hakyemez B, Yildirim N, Taskapiliogle O, Erdogan C, Aker S, Yilmazlar S, Parlak M: Intracranial myeloid sarcoma: conventional and advanced MRI findings. Br J Radiol 80: e109e112, 2007

6) Lee B, Fatterpekar GM, Kim W, Som PM: Granulocytic sarcoma of the temporal bone. AJNR Am J Neuroradiol 23: 1497-1499, 2002

7) Lichtman MA, Rowe JM: Hyperleukocytic leukemias: rheological, clinical, and therapeutic considerations. Blood 60: 279-283, 1982

8) Liu PI, Ishimaru T, McGregor DH, Okada H, Steer A: Autopsy study of granulocytic sarcoma (chloroma) in patients with myelogenous leukemia, Hiroshima-Nagasaki 1949-1969. Cancer 31: 948-955, 1973

9) Nishimura S, Kyuma Y, Kamijo A, Maruta A: Isolated recurrence of granulocytic sarcoma manifesting as extra- and intracranial masses. Case report. Neurol Med Chir (Tokyo) 44: 311-316, 2004

10) Pileri SA, Ascani S, Cox MC, Campidelli C, Bacci F, Piccioli M, Piccaluga PP, Agostinelli C, Asioli S, Novero D, Bisceglia M, Ponzoni M, Gentile A, Rinaldi P, Franco V, Vincelli D, Pileri A Jr, Gasbarra R, Falini B, Zinzani PL, Baccarani M: Myeloid sarcoma: clinico-pathologic, phenotypic and cytogenetic analysis of 92 adult patients. Leukemia 21: 340-350, 2007

11) Struhal W, Oberndorfer S, Lahrmann H, Lindeck-Pozza E, Hess B, Nussgruber V, Pohnl R, Dobner T, Grisold W: Myeloid sarcoma in the central nervous system: case report and review of the literature. Acta Clin Croat 47: 19-24, 2008

12) Suzer T, Colakoglu N, Cirak B, Keskin A, Coskun E, Tahta K: Intracerebellar granulocytic sarcoma complicating acute myelogenous leukemia: a case report and review of the literature. J Clin Neurosci 11: 914-917, 2004

13) Törnebohm E, Lockner D, Paul C: A retrospective analysis of bleeding complications in 438 patients with acute leukaemia during the years 1972-1991. Eur J Haematol 50: 160-167, 1993

14) Tsimberidou AM, Kantarjian HM, Estey E, Cortes JE, Verstovsek S, Faderl S, Thomas DA, Gracia-Manero G, Ferrajoli A, Manning JT, Keating MJ, Albitar M, O’Brien S, Giles FJ: Outcome in patients with nonleukemic granulocytic sarcoma treated with chemotherapy with or without radiotherapy. Leukemia 17: 1100-1103, 2003

15) Tsimberidou AM, Kantarjian HM, Wen S, Keating MJ, O'Brien S, Brandt M, Pierce S, Freireich EJ, Medeiros LJ, Estey E: Myeloid sarcoma is associated with superior eventfree survival and overall survival compared with acute myeloid leukemia. Cancer 113: 1370-1378, 2008

16) Verma D, Kantarjian H, Faderl S, O’Brien S, Pierce S, Vu K, 
Freireich E, Keating M, Cortes J, Ravandi F: Late relapses in acute myeloid leukemia: analysis of characteristics and outcome. Leuk Lymphoma DOI:10.3109/10428191003661852, 2010

17) Widhalm G, Dietrich W, Mullauer L, Streubel B, Rabitsch W, Kotter MR, Knosp E, Roessler K: Myeloid sarcoma with multiple lesions of the central nervous system in a patient without leukemia. J Neurosurg 105: 916-919, 2006

18) Xu Q, Wang $M$, You $Q$, Wang $H$, Ye K, Zhan R, Zhou Y: Isolated recurrence of granulocytic sarcoma. Two case reports. Neurol Med Chir (Tokyo) 49: 611-615, 2009

19) Yamauchi K, Umeda Y: Symptomatic intracranial haemor- rhage in acute nonlymphoblastic leukaemia: analysis of CT and autopsy findings. J Neurol 244: 94-100, 1997

20) Yamauchi K, Yasuda M: Comparison in treatment of nonleukemic granulocytic sarcoma. Report of two cases and a review of 72 cases in the literature. Cancer 94: 1739-1746, 2002

Address reprint requests to: Mineko Murakami, MD, Department of Neurosurgery, Teikyo University, Chiba Medical Center, 3426-3 Anegasaki, Ichihara, Chiba 299-0111, Japan. e-mail: mimurakami-nsu@umin.ac.jp 